\title{
EINE ÄSTHETIK DER GRENZE ALS MASSVOLLES GUTES UND SCHWELLE ZUM ANDEREN — DIE PRAXIS DER FREUNDSCHAFT BEI IVAN ILLICH
}

\author{
Isabella Bruckner
}

UDK 1 Illich, I.

177

27-444.2

Der Begriff der Grenze bzw. der »Obergrenze« ist in den letzten Jahren auf verschiedenste Weise immer wieder zentral geworden; sei es in Verbindung mit den Migrations- und Fluchtbewegungen im demographischen Bereich, sei es das Höchstvolumen kapitalen Einkommens betreffend, sei es im Hinblick auf die Schadstoff-Belastung von Boden, Wasser und Atmosphäre. Insofern ist der Aufruf zur Be-grenzung in aktuellen politischen, wirtschaftlichen und ökologischen Diskussionen ein vielfältiger und oft gehörter. Der 1926 in Wien geborene Grenzgänger Ivan Illich weist mit seinem Beitrag »Philosophische Ursprünge der grenzenlosen Zivilisation « ${ }^{1}$ jedoch darauf hin, dass dieser Begriff der Grenze - gleichsam wie die damit einhergehende Form der Weltwahrnehmung — selbst ein geschichtlich gewordener ist.

Ivan Illich, der vor allem in den 1980er-Jahren im Bereich der Pädagogik und in den Sozialwissenschaften breitere akademische sowie öffentliche Aufmerksamkeit erlangte, stellt in seinen gesellschaftskritischen Schriften große Institutionen der westlichen Moderne in den Mittelpunkt, so u.a. die Pflichtschule, das Medizinsystem, die Entwicklungshilfe oder das moderne Verkehrs- und Transportsystem. Illich erweist deren »Kontraproduktivität«, indem er ihre destruktiven Wirkweisen aufzeigt, sobald solche Einrichtungen und Werkzeuge im Allgemeinen ein gewisses kritisches Maß, eine bestimmte Größe überschreiten. ${ }^{2}$ Im Kontext dieser Gesellschafts- und Institutionenkritik beschäftigte er sich zudem mit einer historischen Rekon-

* Karl-Franzens-Universität Graz, Katholisch-Theologische Fakultät, Institut für Fundamentaltheologie

1 Illich, Ivan: »Philosophische Ursprünge der grenzenlosen Zivilisation«, in: Weizsäcker, Ernst Ulrich von: Grenzen-los? Jedes System braucht Grenzen - aber wie durchlässig müssen diese sein? Basel: Springer 1997, S. 202-211.

2 »Unter Kontraproduktivität, also 'zweckwidriger Wertschöpfung', verstehe ich die Tatsache, daß jenseits gewisser Schwellen eine Institution die Mehrzahl ihrer Klienten von genau jenem Ziel entfernt, zu dem sie gestaltet und finanziert worden ist.« (Illich, Ivan: Entschulung der Gesellschaft. Eine Streitschrift. München: C.H. Beck 62013, S. 12.) 
struktion des Konzeptes der Grenze sowie des Empfindens von maßvoller Stimmigkeit und Andersheit, was ich im Folgenden kurz erläutern möchte. Hierzu werde ich ausgehend von oben genanntem Text kurz auf seine Rekonstruktion der Verlustgeschichte der beiden letzteren Momente eingehen und (weitere Schriften aufgreifend) deren Auswirkungen, besonders hinsichtlich einer möglichen Ethik und Ästhetik, bedenken. Dabei werde ich in besonderer Weise auf die Zentralität der beiden Momente in Illichs Betrachtung der christlichen Praxis der Freundschaft eingehen.

Illich bemerkt zunächst, dass unter den Begriff »Grenze« heute so grundlegend verschiedene Phänomene wie Mauern, Zäune, nationale Gebietsabsteckungen, Obergrenzen für Verkehrsdichte, Stromverbrauch oder monetäre Schulden, ebenso wie Zellmembranen oder auch Grenzwerte in der Mathematik gefasst werden können. Unabhängig davon, dass dieses so weit ausdehnbare, unkonkrete und unsinnliche »Plastik-« oder »Amöbenwort «, wie Illich es nennt, erst mit Martin Luther seinen Weg in die deutsche Sprache gefunden hatte, könne man den Grenzbegriff der Moderne auch unmöglich auf uns ähnlich erscheinende Phänomene der Antike applizieren.

\section{Das Seiende als natürliches Begrenztes im komplementären Denken}

Denn für Illich lasse sich die Moderne gegenüber früheren Epochen durch ein fundamental neues Empfinden von Welt charakterisieren. Er referiert dabei auf den Verlust einer Aisthesis, eine Art des Wahrnehmens und Empfindens, die für unser heutiges Verständnis kaum mehr zu erspüren ist, die aber alle von Illich untersuchten Kulturkreise prägte. ${ }^{4}$ Es geht ihm dabei um ein Wahrnehmen von Welt, welches jegliches Seiende als mit einem konstitutiv Anderem grundsätzlich in Beziehung stehend betrachtete. Die Identität des einen lag jeweils in der komplementären Andersheit des Anderen begründet. Ein Seiendes, so Illich, konnte nicht isoliert betrachtet werden, sondern stets nur in seiner Beziehung zum es bedingenden Anderen, ohne welches es nicht zu existieren vermocht hätte. Um diese Auffassung zu unterstreichen, bringt Illich auch etymologische Überlegungen ins Spiel: »Kosmos« bezeichnete ursprünglich mehr als bloße»Ordnung« der Welt; das Wort leite sich vom griechischen Wort »chosmein« her, was er als »einander dem Amerikanischen von Ruth Cremerius. Hamburg: Hoffmann und Campe 1988, S. 122. 
gegenüber aufreihen $\aleph^{5}$ übersetzt. Damit verweist er auf eine fundamentale komplementäre Dualität, die durchgängig das Denken, Empfinden und Wahrnehmen geprägt haben soll. Illich erblickt darin das präreflexive Empfinden einer Bezogenheit oder Entsprechung alles Seienden ${ }^{6}$ und verwendet dafür häufig den Ausdruck »dissymmetrische Proportionalität«:

Wenn ich über die Geschichte der Proportionalität nachdenke, sehe ich, dass nichts existieren kann, ohne dissymmetrisch proportional zu etwas anderem zu sein, und dass diese dissymmetrische Proportionalität der Grund ist für beider Existenz. [...] Nichts ist einfach, was es ist, sondern nur deswegen, weil es convenit, weil es passt, weil es in Harmonie mit etwas anderem ist. ${ }^{7}$

Gemäß einer solchen Weltwahrnehmung entsprach z.B. die Anzahl der Grashalme jener der Sterne im Himmel; das Auge hatte Sinn nur deshalb, weil es mit der Sonne in einer wesenskonstitutiven Beziehung stand; der Mann existierte nur als das Andere zur Frau; und der Pilz stand im Garten, insofern er in der richtigen Dosierung als Heilmittel bei Nierenleiden diente. Alles Seiende stand somit in einer wesenskonstitutiven Bezüglichkeit, in Harmonie. Ohne diese konnte nichts gedacht werden. Das Seiende wurde demnach zum einen als ein grundsätzlich in Beziehung Stehendes gedacht, und zum anderen als durch dieses Bezugskorrelat immer schon Begrenztes - bzw., um es für heutige Ohren positiver zu formulieren: Das Seiende wurde als ein Maßvolles empfunden.

Den Angelpunkt der Bezüglichkeit aber bildete der Mensch. Er galt als das Maß der Dinge, der Mikrokosmos, in dem sich der Makrokosmos spiegelte. Die menschlichen Gemeinschaften versuchten in ihrem jeweiligen ethos, diese vielfachen Analogien und Proportionen wahrzunehmen und sich auf diese einzuschwingen. Dieses gemeinschaftliche Empfinden dessen, was »passt«, was »harmoniert«, sowie der darauf basierenden lokalen Praxis, versteht Illich unter dem Begriff der »Kultur«. Insofern stellten die »natürlichen« Begrenzungen und Beziehungen für Illich immer schon gemeinschaftlich über die Kultur, d.h. über die »zweite Natur«, vermittelte Topologien dar. Eine rein objektive natürliche Ordnung an sich, gibt es in seinen Augen demnach nicht. Erst durch das Wahrnehmen des Menschen wird die Welt zum Kosmos. Gemäß der je kontingenten Lebensbedingungen

5 Vgl. Illich:Philosophische Ursprünge der grenzenlosen Zivilisation, S. 207.

6 Michel Foucault, der im selben Jahr wie Illich geboren wurde und dessen Archäologie der Institutionen und noetischen Selbstverständlichkeiten der Moderne sehr an dessen Arbeitsweise erinnert, analysiert die besagten Weisen des Denkens ausführlich in seinem umfangreichen Werk Die Ordnung der Dinge. Eine Archäologie der Humanwissenschaften. (Les mots et les choses.) Aus dem Französischen von Ulrich Köppen. Frankfurt am Main: Suhrkamp 1971.

7 Illich, Ivan: In den Flüssen nördlich der Zukunft. Letzte Gespräche über Religion und Gesellschaft mit David Cayley. Aus dem Englischen von Sebastian Trapp. München: C. H. Beck 2006, S. 223. 
bildeten sich deshalb auch ganz unterschiedliche soziale Lebensformen heraus, deren Gemeinsamkeit Illich aber in der Form, d.h. in diesem Sich-Einstimmen auf die maßvolle Harmonie des Kosmos, sieht. Jede Gemeinschaft entwickelte einen je eigenen sensus communis, dem entsprechend etwas passend und mit seinem begrenzten telos in Harmonie stehen konnte. Das Gute der antiken Ethik war demnach stets ein Maßvolles, Begrenztes.

\section{Der Verlust des maßvollen Guten}

Dieses durch anderes angemessen begrenzte Gute ging jedoch in der Neuzeit seiner Konsistenz verlustig. Illich verortet erste dahingehende ästhetische Brüche bereits im 13. Jahrhundert, reflektiert in der mönchischen Theologie des Hochmittelalters. Er hebt hierbei auf die explizite Herausbildung des Kontingenzgedankens ab, welchen er in der franziskanischen Tradition auf die Spitze getrieben sieht. Denn in der spät-antiken christlichen Theologie fand sich das Seiende nicht mehr nur in eine innerkosmische Proportionalität eingebunden, sondern darüber hinaus als zur Gänze vom gnadenhaften Willen eines transzendenten Gottes abhängig. Der Wille Gottes bildete in diesem Sinne sozusagen die »vertikale« Entsprechung eines jeglichen Seienden im Gegensatz zur innerweltlichen »horizontalen« Komplementarität der Seienden untereinander. Doch in der Theologie des 13./14. Jahrhunderts beginnt nach Illich dieser Kontingenzgedanke an voluntaristische Willkürlichkeit zu grenzen, obwohl er selbst bei Wilhelm von Ockham noch den Bezug auf ein Jenseitiges (den Willen Gottes), welches dem Diesseitigen seine Begrenzung gab, verankert sieht. ${ }^{8}$ Erst im Denken René Descartes‘, Isaac Newtons und Gottfried Wilhelm Leibniz‘ verlor sich laut Illich das Jenseits als komplementäres, begrenzendes Anderes ${ }^{9}$ - seine raison d'être und Identität fand jedes Wesen seitdem in seiner eigenen Natur begründet. »Die Na-

8 Die Diskussion zur franziskanischen Ethik ist heute sehr umstritten. Ich umgehe sie an dieser Stelle und halte mich strikt an Illichs eigene Einschätzung (vgl. Illich: In den Flüssen, S. 92). Zur genaueren Debatte siehe jedoch u.a. Schröcker, Hubert: Das Verhältnis der Allmacht Gottes zum Kontradiktionsprinzip nach Wilhelm von Ockham. Berlin: Akademie Verlag 2003, S. 100-107; Beckmann, Jan Peter: Wilhelm von Ockham. München: C.B. Beck 1995, S. 153-156; Crowe, Michael Bertram: The Changing Profile of the Natural Law. The Hague, Netherlands: Martinus Nijhoff 1977, S. 194-203.

9 »Grenze wurde zum festen Begriff in der Übersetzung des Leibniz‘schen InfinitesimalKalküls. Er bezeichnet den Grenzwert einer konvergenten Folge. Überraschenderweise hat gegen Ende des 18. Jhds dieser Einsatz des Wortes zur gelehrten Übersetzung den Gebrauch des Ausdrucks Grenze in der Umgangssprache beeinflusst, denn nun wird bei seiner Verwendung zunehmend von einem jenseits der Grenze Liegenden abgesehen. [...] Für Leibniz 'grenzen Monaden nicht an': jede ist durch sich selbst limitiert. Sie ist die Grenze ihres inneren Aufbaus. (Illich: Philosophische Ursprünge der grenzenlosen Zivilisation, S. 206.) Vgl. auch Illich: In den Flüssen, S. 94, 163. 
tur wird zur 'drüben-losen Selbst-Begrenzung “ ${ }^{10}$, d.h. ohne diese vertikale und horizontale Komplementarität gedacht.

\section{Der Verlust von Genus und konstitutiver Andersheit}

Zunächst resultierte aus diesem Verlust des maßvollen Guten die Transformation der immanenten Teleologie zu einem Entwicklungskonzept des maßlosen, ständigen Über-sich-hinaus-Strebens. ${ }^{11}$ Zudem ging in Illichs Augen mit dem Verlust des proportionalen Denkens jedoch auch das Schwinden einer bestimmten Empfindung von Andersheit einher. Das proportionale Denken basierte auf der Ästhetik einer als fundamental erfahrenen Differenz. Die dissymmetrische Proportionalität konstituierte sich aus der notwendigen gegenseitigen Bezogenheit zweier Anderer aufeinander.

Ausführlicher versucht Illich dies am Beispiel des Geschlechterverhältnisses zu zeigen: ${ }^{12}$ In vorindustriellen Gesellschaften fand diese Differenzerfahrung ihre soziale Ausgestaltung u.a. in den unterschiedlichen Lebenswelten von Mann und Frau. Diese komplementären geschlechtsspezifischen sozialen Sphären versucht Illich unter dem Begriff Genus zu fassen. Bis ins hohe Mittelalter habe Genus ungebrochen das Empfinden, Denken und Handeln der Menschen in Europa geformt sowie in gleicher Weise bei allen anderen Völkern der verschiedenen Erdteile, deren Schriften er durchforstete. Zumindest Reste davon finden sich auch heute noch in den Kulturen verankert. ${ }^{13}$ Genus durchformte auf lokal völlig unterschiedliche Weise alle Lebensausdrücke, wie z.B. Gestik, Mimik, Sprache in Wortwahl und Intonation, die jeweiligen Arbeitsbereiche, den spezifischen Umgang mit Werkzeug, den vorgesehenen Ritualen usw. der Männer und Frauen. Die Welt der

10 Illich: Philosophische Ursprünge der grenzenlosen Zivilisation, S. 208.

11 Hans Schelkshorn untersucht diese Dynamik in den verschiedensten gesellschaftlichen Bereichen und konstatiert: »Der Versuch, die Moderne als ein komplexes Spiel von Entgrenzungen zu deuten, eröffnet zugleich den Blick auf eine spezifische 'Logik' bzw. 'Dialektik' neuzeitlichen Denkens. Da in jeder Überschreitung von Grenzen unvermeidlicherweise neue Grenzen gesetzt werden, bildet sich [...] eine Steigerungsdynamik, insofern die neuen Grenzen neue Überschreitungen provozieren.« (Entgrenzungen. Ein europäischer Beitrag zum philosophischen Diskurs über die Moderne. Weilerswist, Deutschland: Velbrück Wissenschaft 2009, S. 599.) Anders als Illich lenkt er seinen Fokus jedoch vor allem auf diverse Um- und Aufbrüche (insbesondere die Geographie, Kosmologie, Epistemologie und Anthropologie betreffend) im Denken der Renaissance.

12 Siehe hierzu sein kontrovers diskutiertes Buch Genus. Zu einer historischen Kritik der Gleichheit (= Beck'sche Reihe 1105). München: C.H. Beck'sche Verlagsbuchhandlung 21995.

13 Siehe hierzu Endnote 88: »In der sozialen Wirklichkeit kann Genus schwinden, verblassen, von sexuellen Polarisationen oder Unisex überlagert werden — verschwinden kann Genus wohl nicht.« (Illich: Genus, S. 198.) 
Frauen war eine andere als die der Männer. Sie bewohnten zwar teilweise denselben Raum, formten und gestalteten diesen aber aufgrund ihrer divergierenden Körper und Tätigkeiten unterschiedlich. Die Welt der Frauen war für die Männer eine fremde (und vice versa), und trotzdem oder gerade deshalb aufgrund der Komplementarität beider Welten für sie unabdingbar; wesentlich war der Lebensform von Genus also die gegenseitige Verwiesenheit auf den Anderen eingeschrieben.

Deutlich versucht Illich davon die moderne Kultur des Sexus zu unterscheiden. Dem Sexus liegt die Idee eines geschlechtslosen Individuums zugrunde, welches sozial in keine konkrete kulturelle Sphäre eingebettet ist und dem erst sekundär physische und psychische sowie soziale Eigenschaften zugeschrieben werden. René Descartes' res cogitans und Gottfried Wilhelm Leibniz‘ Monade sind als neutrale Subjekte zu verstehen — die Grundlage für die Idee von »dem Menschen«, dem vor aller historischen, kulturellen Eingebundenheit allgemeine Eigenschaften und Bedürfnisse zugeschrieben werden.

Am Genus zeigt sich in seinen Augen deutlich die vorindustrielle Ästhetik, basierend auf dem Prinzip wesenhafter Andersheit bei gleichzeitiger Bezogenheit. Genus bezeichnet nicht einfachhin ein Set an Eigenschaften, das beliebig abgelegt werden kann; in Genus wird man hineingeboren und dieser symbolische Kontext prägt die gesamte Weltwahrnehmung. Frau-Sein und Mann-Sein sind im Horizont einer solchen Ästhetik keine sekundären Eigenschaften, von denen man sich als neutrale Person einfach distanzieren könnte. Nach dem Verlust, in einer Kultur des Sexus, werde Andersheit hingegen nur mehr hinsichtlich zufälliger, äußerer Merkmale konstatiert. Illich erkennt in jener Entwicklung zwei grundsätzlich verschiedene Arten »Dualität« und Andersheit zu verstehen:

I became increasingly convinced that the deepest change I could observe between then and now, between a prescientific, preindustrial [...] past, and our present worldview [...], was the transition from one type of duality to another. It is quite clear that "two « can be conceived of in two different ways. When I say one, two can mean primarily, emotionally, conceptually the other, or it can mean one more of the same. [...] It seems to me that in all preliterate societies, or prealphabetic Western societies I can study, the first way of conceiving duality shaped the depths of consciousness. There is me and there is the other; there is the microcosmos and there is the macrocosmos; there is this world and the other world; here are the living and there are the dead; and, in the most profound sense, I am a man, and these others, women, are shaded for me, muted for me, other for me. [...] [O]therness, even at the height of intimacy, was what gave ultimate consistency to what we today call consciousness, to being here. With the seventeenth century [...] the human being, the self, the individual, became the model of our thinking. And then an entirely new way of seeing the other came into existence: he is an other with a black skin; the post-Cartesian inside is a special zone within a general space; [...]. The tension between dissymmetric 
complementarities was collapsed into an a priori abstract notion, which then finds accidental distinctions. ${ }^{14}$

Diese gesellschaftliche und ästhetische Transformation ist unrevidierbar. Der Verlust des Komplementaritätsempfindens (der "großen Tradition« wie Illich dies auch immer wieder nennt ${ }^{15}$ ) — und damit verknüpft der Verlust von Genus - resultierte insofern in der Herausbildung abstrakter Raum- und Zeiteinheiten. Vor diesem Verlust korrelierte der Komplementarität im Bereich der Seienden ein ebenso duales bzw. komplementäres Raum-Verständnis. Bei dem Innen und Außen eines Zaunes handelte es sich dabei nicht um den qualitativ gleichen, neutralen Raum, der nur zufällig durch Holzlatten voneinander abgegrenzt war. Ein Zaun war keine einfach willkürlich gesetzte Linie auf einer qualitativ gleichwertigen Fläche von Quadratmetern, sondern bildete die Scheide zweier grundsätzlich verschiedener, doch konstitutiv aufeinander bezogener Sphären von Innen und Außen. Wie Männer und Frauen Raum und Zeit beschrieben, artikulierte sich nicht in abstrakten Wertmaßstäben, die von jeder konkreten Empfindung abgekoppelt gewesen wären, sondern in Vergleichen und Relationen aus der alltäglichen Lebenswelt der Frau oder des Mannes in der jeweiligen Kultur. Auch die Wahrnehmung von Raum und Zeit gestaltete sich insofern als genus-spezifisch und sozial vermittelt.

Kurzum: Was Illich, in meinen Augen, unter anderem damit zu äußern versucht, ist seine Beobachtung des Verlustes eines sozial vermittelten symbolischen »Zuhause-Seins« im Kosmos, der als ein Zusammenstimmen grundsätzlich verschiedener (und insofern begrenzter, darin aber gut seiender!) Teile verstanden und empfunden wurde. Ethik und Kosmologie konnten in dieser Ästhetik nicht voneinander getrennt werden, sondern basierten auf demselben Prinzip der proportionalen Harmonie. Gegenstände, Räume und Zeiten ebenso wie Tätigkeiten und soziale Rollen waren damit stets symbolisch und narrativ eingebettet, korrelierten jedoch allesamt mit dem sinnenhaften Empfinden des darin vermittelten Komplementaritätsprinzips.

\section{Die freundschaftliche Beziehung des Samariters als Krönung der Komplementarität}

Interessanterweise charakterisiert Illich jedoch die Freundschaftsbeziehung als eine besondere Form der Komplementarität, in der dieses Empfinden der at the Lecture Series "Conversations: The Legacies of Ivan Illich” at Pitzer College, Claremont (California, USA), March 26-March 28, 2004, verfügbar unter: http://www.pudel. uni-bremen.de/pdf/robert_Clar04_demise_philia_en.pdf [24.11.2017]. 
konstitutiven Andersheit des Gegenübers immer noch aufzublitzen vermag. ${ }^{16}$ Die Andersheit spielt in dem, was Illich Freundschaft nennt, eine wesentliche Rolle — die »Ehrfurcht vor dem Geheimnis des Ganz-Anders-Seins « ${ }^{17}$ bildet für ihn gar die Grundlage echter Freundschaft — und letztlich scheint es ihm in seinem Leben und Schreiben im Wesentlichen um die Bewahrung und Verwirklichung dieser Beziehung zu gehen. ${ }^{18}$ In seinem Denken über die Freundschaft ist Illich klar biblisch sowie klassisch philosophisch und theologisch geprägt. Grundsätzlich ist jedoch zu bemerken, dass er bei der Freundschaft, nach welcher er strebt, vom Glauben an die Inkarnation ausgeht. Für ihn resultiert aus der Inkarnation zum einen eine völlig neue Form des Liebens, zum anderen eine neue und intensive Art der Wahrnehmung und Empfindung von Welt, Selbst und dem anderen. ${ }^{19}$ Mit der Inkarnation verbindet er insbesondere die gewandelte Bedeutung des Fleisches, eine neue Ästhetik der sinnlichen, empfindsamen und verletzlichen Körperlichkeit des Menschen. Insofern spricht er von einer »geheimnisvolle[n] neue[n] Herrlichkeit, Fülle, phänomenologische[n] Dichte, die der Körper unter dem Einfluss des Christentums annimmt ${ }^{20}$ und dem »eine meta-physische Qualität zu[kommt], die mehr als nur eine Laune des Augenblicks [ist]«.

Illich illustriert die im Christentum historisch feststellbare neue Form der Beziehung oftmals an der Erzählung vom Samariter. ${ }^{21}$ In seiner Zuwendung zu dem am Straßenrand liegenden Juden begeht der zufällig vorbeikommende Samariter einen regelrechten Verrat an seiner Gemeinschaft. Er übertritt damit die damals geltenden Grenzen seines ethos und geht in Illichs Lesart ein ungeheures Wagnis ein. Er bewegt sich dabei in einem Raum der Unbestimmtheit, der weder durch allgemeine Normen noch durch gängige Praxis vorab strukturiert ist. Der Samariter reagiert und handelt, gemäß dem Text des Evangeliums, allein aufgrund der Rührung seines splanchnas, seines sensiblen Inneren. ${ }^{22}$ So wie dies auch in Bezug auf Jesus und JHWH selbst immer wieder anklingt, ${ }^{23}$ folgt der Samariter der innerlichen Ergriffenheit, die ihn beim Anblick oder aufgrund des Schreis des Juden körperlich trifft. aus dem Münchener NT: »Ein Samariter aber, der unterwegs war, kam zu ihm, und sehend (ihn), erbarmte er sich«. Illich verwendet statt »er erbarmen sich« gerne den Ausdruck der Lutherbibel: »er jammerte ihn«. Vgl. z.B. Ex 34,6; Jes 49,15; Mk 6,34. 
Die Ethik, die Illich hier andenkt, gründet sich somit einerseits auf eine gesteigerte Affektivität und Berührbarkeit, andererseits auf das Prinzip geschenk- bzw. gnadenhafter Freiheit in Verantwortung. Eine derart geschaffene Beziehung, die über alle »natürlichen« Positionierungen hinweg, allein durch das freie Füreinander-Dasein-Wollen zweier anderer besteht, stellt im mittelmeerischen antiken Kulturraum laut Illich eine Neuheit dar. ${ }^{24}$ Als solche bedeute sie zumindest idealiter aber keine Negierung, sondern eher die »Krönung ${ }^{25}$ der auf dem harmonikalen Guten basierenden Ethiken. Illich betrachtet die frei geschenkte Beziehung des Samariters mit dem Juden als die gnadenhafte (weil nicht schon vorab durch Blutsbande verpflichtende oder auf irgendeine Weise zweckdienliche) Aufhebung ${ }^{26}$ der wesenhaften Komplementarität des Menschen und damit zugleich der gesamten proportionalen Schöpfung. Die natürliche Bezüglichkeit wird dabei in einem (ungesicherten) Raum der Freiheit verwirklicht. ${ }^{27}$

Diese Denkweise steht in der scholastischen Tradition des gratia naturam perficit ${ }^{28}$ — die Gnade vervollkommnet die Natur:

Der Ruf der Nächstenliebe, agape, den der Samariter hörte, zerstört die Proportionalität nicht, sondern hebt sie auf eine neue Stufe, die früher nicht denkbar war. Das geht über Plato und Aristoteles ebenso hinaus wie über die griechischen Mysterien. Es besagt, dass dein telos, deine Bestimmung, das Ziel, die »Lock-Ursache« deines Daseins, in einem anderen liegt, den du frei wählst. ${ }^{29}$

Das klassische Verhältnis von Tugend und Gemeinschaft wird damit in der christlichen Ethik verkehrt, gerade darin aber gekrönt. Für Platon und Aristoteles bildete die (politische oder intellektuelle) Tugend als solche das vorrangige telos des Strebens, und die freundschaftliche Gemeinschaft eröffnete sich allein als Möglichkeit für jene, die diese Tugendhaftigkeit der Polis auch verwirklichen konnten (d.h. eine Beziehung unter Gleichen).

24 Vgl. Illich: In den Flüssen, S. 76f. Illich bezieht sich hierbei auf seinen »großen Lehrer« Gerhard Ladner, der in seinem Buch The Idea of Reform: Its Impact on Christian Thought and Action in the Age of the Fathers (Cambridge, Mass. 1959) die neuartige Idee von Reform, die im Christentum aufkam, und als Bekehrung bzw. Selbst-Erneuerung aufgefasst wurde, untersucht.

25 Vgl. das Kapitel »Die krönende Herrlichkeit« in Illich: In den Flüssen, S. 220-225.

26 Aufhebung wird hier im dreifachen Sinne G.W.F. Hegels verstanden als Negierung des bestehenden Verhältnisses (1), Erlangung einer neuen höheren Ebene (2), wobei jedoch die negierten Momente nicht einfachhin zurückgelassen werden, sondern als notwendig vorangehende Stufen bewahrend mitaufgehoben werden (3), wodurch diese in einer gewissen Validität erhalten bleiben.

27 Vgl. In den Flüssen, S. 231f.

28 Vgl. Milana, Fabio: Ivan Illich, Breaking the silence, 11.1.2014, verfügbar unter: http:// backpalm.blogspot.co.at/2014/01/ivan-illich-breaking-silence.html\#more [23.11.2017].

Illich: In den Flüssen, S. 223. 
Das vorrangige telos des Menschen, den Christus verkörpert und der sich zu dieser neuen Form der Beziehung berufen weiß, liegt nun im anderen Menschen selbst begründet. Dieses kommt dem Christen vom Gegenüber her, in dessen Anruf, Willen und Wunsch, in dessen Blick und Stimme entgegen. Die christliche Beziehung gestaltet sich insofern wesentlich als eine des Gehorsams ${ }^{30}$ (im Sinne des Hörens-auf) in der Freiheit von kulturellen, ständischen und anderweitigen Begrenzungen und für den zufällig begegnenden anderen.

Illich betont jedoch, dass in der Beziehung, die zwischen dem Samariter und dem Juden entsteht, die Unterschiedenheit der einander Begegnenden und deren differente kulturelle Formung nicht negiert wird. Sie begegnen sich als vorgängig andere, Fremde, die als solche einander nichts schulden. Es gibt in dieser Begegnung keine gemeinsame Tugend, kein gemeinsames ethos, keinen gemeinsamen Boden. Diese uneinholbar differente Positionalität und Prägung des anderen wird in der Freundschaftsbeziehung nicht zerstört. Jedoch kann aus dieser fragilen Bindung sodann ein neuer ethos entspringen, d.h. eine neue Form des gemeinsamen Lebens - unvorhergesehen und allein darauf basierend, was diese jeweiligen anderen einander und was sich ihnen in ihrer Beziehung schenkt. ${ }^{31}$

\section{Eine Ethik und Ästhetik der Freundschaft als Refugium fundamentaler Alterität}

Illich charakterisiert somit die Andersheit im Kontext von Genus als eine, die den anderen den eigenen Kategorien und Denkschemata grundlegend entzieht (»[...] in the most profound sense, I am a man, and these others, women, are shaded for me, muted for me, other for me«) - im Gegensatz zu einer Andersheit, die sich an partikulären bzw. sekundären positiv fassbaren Äußerlichkeiten (»he is an other with a black skin«) bei grundlegender Gleichheit (»the human being«) festmachen lässt. Eine christliche Ethik, die sich auf die Erzählung des barmherzigen Samariters stützt, lädt dazu ein, diese Grenzen (sowohl »heimatliche«, d.h. kulturell gewachsene Eigenheiten, die konkrete »zweite Natur«, als auch zufällig erworbene Unterschiede oder — wie in heutiger Zeit üblich — sich gegebene Identitäten) zwar wertzuschätzen, sie aber immer wieder auf den Anderen hin zu überschrei-

30 Gronemeyer, Marianne: Freiheit und Gehorsam. Vortrag gehalten anläßlich des 2. Symposioms zum Tod von Ivan Illich 2003 in Bremen, verfügbar unter: http://www.pudel. uni-bremen.de/pdf/Gronemeyer03fr.pdf [21.11.2017].

31 Vgl. Trapp, Sebastian: Der Ethnos der Freundschaft. Vortragsmanuskript für das »Symposium für Ivan Illich zum Abschied«, 7./8. Februar 2003, Universität Bremen, verfügbar unter: http://www.pudel.uni-bremen.de/pdf/Trapp_Sym_02_03_dt.pdf [23.11.2017], S. 3f. 
ten und sie ihm in einem Akt der Freiheit zu (er-)öffnen. Jene Grenze des Gegenübers (seine grundsätzliche Differenz und Uneinholbarkeit, basierend auf seiner endlichen Perspektivität und seiner Freiheit) hat eine christliche Ethik der Freundschaft dahingegen stets zu wahren. Dadurch bleibt der Andere wesentlich unverfügbar. Dies versucht Illich mit dem Begriff der Überraschung auszudrücken. ${ }^{32}$ Mit der Haltung des Sich-vom-Anderenüberraschen-lassens ist ein Ablassen von jeglicher Bemächtigung des Anderen impliziert. Den Anderen nicht (schon vorab) in die eigenen Kategorien einordnen zu wollen, oder ihn durch äußerliche Maßstäbe zu bestimmen, verlangt Vertrauen in das, was der Andere selbst von sich offenbart. ${ }^{33}$

In einem Horizont der Überraschung schenkt sich der Andere immer wieder neu, immer wieder anders. Insofern wird die Forderung verständlich, »einem alten Freund wie einem Fremden in die Augen sehen zu können und einem Fremden wie einem alten Freund « ${ }^{34}$. Deshalb ist es auch möglich im Kontext der Samariter-Erzählung von Freundschaft zu sprechen, obwohl diese in der Regel als das Paradebeispiel barmherzigen Handelns angeführt. Doch in dieser in Freiheit gewagten Barmherzigkeit, in dieser zufälligen persönlichen Begegnung, steckt bereits wie in einem Samenkorn das Potential für eine lang anhaltende intensive Beziehung. ${ }^{35}$

32 »Obedience in the biblical sense means unobstructed listening, unconditional readiness to hear, untrammeled disposition to be surprised. It has nothing to do with what we call obedience today, something that always implies submission, and ever so faintly connotes the relationship between ourselves and our dogs. When I submit my heart, my mind, my body come [sic!] to be below the other. When I listen unconditionally, respectfully, courageously with the readiness to take in the other as a radical surprise, I do something else. I bow, bend over towards the total otherness of someone.« (Illich, Ivan: The Educational enterprise in the Light of the Gospel, Chicago Nov 13th 1988, verfügbar unter: http://www.davidtinapple.com/illich/1988_Educational.html [23.11.2017]. [Herv. I.B.] Auf Französisch erschienen: »L'entreprise éducative actuelle vue par le marginal à la lumière de l'evangile«, in: La perte des sens, a.a.O.) Vgl. auch Illich, Ivan: »Die Schule als heilige Kuh«, in: Klarstellungen. Pamphlete und Polemiken (= Beck'sche Reihe 1151). Aus dem Englischen von Helmut Lindemann. Mit einer Einleitung von Erich Fromm. München 1996, S. 13-25, hier: S. 24.

33 Hierin könnte man die Verwirklichung der theologischen Tugend des Glaubens erkennen, die, sofern man die Inkarnation ernst nimmt, ebenso ins Intersubjektive gewendet werden muss. In diesem Sinne der aufs Engste verknüpften Gottes - und Nächstenliebe geht es Illich, m. E., stets um eine »Bekehrung und Hinwendung zum Anderen, dem Freund, und das ist selbstverständlich der Mensch gewordene Gott, den ich aber in dem Menschen erkenne, der mir in diesem Augenblick sein Angesicht zeigt« (Illich: In den Flüssen, S. 106). Trapp: Der Ethnos der Freundschaft, S. 4.

35 »Was zwischen dem Juden und dem Samariter geschieht, ist wie ein Samenkorn. Wenn es heranwächst, wird es gerüttelt und vielleicht wird sein Stengel sogar gebrochen, und es wird nie zur Blüte kommen. Woran wir uns festhalten, ist das Samenkorn. Nicht alle Freundschaften sind schön oder herrlich oder voll entwickelt.« (Illich: In den Flüssen, S. 209f.) 


\section{Das Pflegen von Schwellen in einer alteritätslosen Gesellschaft}

Um wirklicher Andersheit und damit dem möglichen Erblühen von Freundschaft in der alteritätsarmen Zeit nach dem Schwund der »großen Tradition" Raum zu schaffen, bedarf es nach Illich zwar keiner Grenzen im heutigen Sinne, aber sehr wohl wirklicher Schwellen - sichtbarer Zwischen-Räume, die den Übergang vom unpersönlichen in einen persönlichen (nicht jedoch »privaten«) Handlungsbereich markieren. Türen und Fenster dienen so weder der Abgrenzung und Abschottung nach außen hin noch fungieren sie als Schranken mit selektiver Durchlässigkeit, sondern sie gewähren vielmehr ein Spiel der Freiheiten in gastfreundschaftlichen Gesten des Einladens, TürÖffnens, Über-die-Schwelle-Geleitens, Platz-Einräumens usw. Illich geht es, wie gesagt, beim Pflegen von »Schwellen, Simsen, Decken ${ }^{36}{ }^{36}$, die qualitativ verschiedene Räume einander gegenüberstellen und die Unterscheidung eines »Außen« von einem »Innen« ermöglichen, nicht um exkludierende Grenzziehungen im Sinne eines »wir« und »die anderen«. Doch benötigt es in seinen Augen diese wahrnehmbaren Übergänge, damit nicht nur ein gleichgültiges Sein-Lassen auf dem neutralen Boden der Öffentlichkeit (bzw. in privater Zurückgezogenheit), sondern ein bewusstes, persönliches (Sich-auf-) den-Anderen-Einlassen statthaben kann. Es braucht »fleischliche« Räume geteilten Miteinanders mit gastfreundlichen, echten Schwellen — nicht nur virtuelle Chatrooms im Cyberspace -, um persönlichen, leibhaftigen Begegnungen und wirklicher Alteritätserfahrung einen neuen Boden zu bieten.

\section{Literaturverzeichnis}

Beckmann, Jan Peter: Wilhelm von Ockham. München: C.B. Beck 1995.

Bruckner, Isabella: Ivan Illich — Freundschaft als Topos christlicher Weltbegegnung (= Diplomarbeit). Wien 2016.

Caley, David: Ivan Illich in Conversation. Toronto: Anansi Press 1992.

Crowe, Michael Bertram: The Changing Profile of the Natural Law. The Hague, Netherlands: Martinus Nijhoff 1977.

Foucault, Michel: Die Ordnung der Dinge. Eine Archäologie der Humanwissenschaften. (Les mots et les choses.) Aus dem Französischen von Ulrich Köppen. Frankfurt am Main: Suhrkamp 1971.

Gronemeyer, Marianne: Freiheit und Gehorsam. Vortrag gehalten anläßlich des 2. Symposioms zum Tod von Ivan Illich 2003 in Bremen, verfügbar unter: http:// www.pudel.uni-bremen.de/pdf/Gronemeyer03fr.pdf [21.11.2017]. 
Illich, Ivan: »Die Schule als heilige Kuh«, in: Klarstellungen. Pamphlete und Polemiken (= Beck'sche Reihe 1151). Aus dem Englischen von Helmut Lindemann. Mit einer Einleitung von Erich Fromm. München 1996, S. 13-25.

Illich, Ivan: Entschulung der Gesellschaft. Eine Streitschrift. München: C.H. Beck 62013.

Illich, Ivan: Genus. Zu einer historischen Kritik der Gleichheit (= Beck'sche Reihe 1105). München: C.H. Beck'sche Verlagsbuchhandlung 21995.

Illich, Ivan: »Philosophische Ursprünge der grenzenlosen Zivilisation«, in: Weizsäcker, Ernst Ulrich von: Grenzen-los? Jedes System braucht Grenzen — aber wie durchlässig müssen diese sein? Basel: Springer 1997, S. 202-211.

Illich, Ivan / Sanders, Barry: Das Denken lernt schreiben. Lesekultur und Identität. Aus dem Amerikanischen von Ruth Cremerius. Hamburg: Hoffmann und Campe 1988.

Illich, Ivan: In den Flüssen nördlich der Zukunft. Letzte Gespräche über Religion und Gesellschaft mit David Cayley. Aus dem Englischen von Sebastian Trapp. München: C. H. Beck 2006.

Illich, Ivan: The Educational enterprise in the Light of the Gospel, Chicago Nov 13th 1988, verfügbar unter: http://www.davidtinapple.com/illich/1988_Educational. html [23.11.2017]. Auf Französisch erschienen: »Ĺentreprise éducative actuelle vue par le marginal à la lumière de l'evangile«, in: La perte des sens. Paris: Fayard 2004.

Ladner, Gerhard: The Idea of Reform: Its Impact on Christian Thought and Action in the Age of the Fathers. Cambridge, Mass.: Harvard University Press 1959.

Milana, Fabio: Ivan Illich, Breaking the silence, 11.1.2014, verfügbar unter: http:/ backpalm.blogspot.co.at/2014/01/ivan-illich-breaking-silence.html\#more [23.11.2017].

Robert, Jean: The demise of the Great Tradition (Lecture note). Lecture given at the Lecture Series "Conversations: The Legacies of Ivan Illich" at Pitzer College, Claremont (California, USA), March 26-March 28, 2004, verfügbar unter: http://www.pudel.uni-bremen.de/pdf/robert_Clar04_demise_philia_en.pdf [24.11.2017].

Schelkshorn, Hans: Entgrenzungen. Ein europäischer Beitrag zum philosophischen Diskurs über die Moderne. Weilerswist, Deutschland: Velbrück Wissenschaft 2009.

Schröcker, Hubert: Das Verhältnis der Allmacht Gottes zum Kontradiktionsprinzip nach Wilhelm von Ockham. Berlin: Akademie Verlag 2003.

Trapp, Sebastian: Der Ethnos der Freundschaft. Vortragsmanuskript für das »Symposium für Ivan Illich zum Abschied «, 7./8. Februar 2003, Universität Bremen, verfügbar unter: http://www.pudel.uni-bremen.de/pdf/Trapp_Sym_02_03_dt.pdf [23.11.2017]. 
Abstract

In the course of his critique on institutions and modern society, the historian and philosopher Ivan Illich seeks to understand how the conception of limitation has changed from antiquity to modernity. Illich speaks about the fading of an aesthetic of proportionality and complementarity, which has framed the perception of beings as well as that of space and time. Within this aesthetic, the experience of fundamental otherness was a constitutive element. The article illustrates Illich's historical analysis and points out its significance for a reflection on the Christian practice of friendship. In the practice of friendship, otherness and complementarity are experienced in a horizon of freedom. In this context, thresholds and visible passages do not function as divisions or mediums of exclusion, but as perceptible markers, which can enable personal action in qualitatively differing spaces.

KEY wORDS: otherness - complementarity — proportionality — limitation — treshold — aesthetics 